\title{
Adaptive Identification and Control Algorithms for Nonlinear Bacterial Growth Systems*
}

\author{
D. DOCHAIN† and G. BASTIN†
}

Simple self-tuning type controllers for nonlinear bacterial growth processes can be effective and their stability can be proven under mild conditions.

Key Words-Adaptive control, fermentation processes, nonlinear systems, parameter estimation.

\begin{abstract}
This paper suggests how nonlinear adaptive control of nonlinear bacterial growth systems could be performed. The process is described by a time-varying nonlinear model obtained from material balance equations. Two different control problems are considered: substrate concentration control and production rate control. For each of these cases, an adaptive minimum variance control algorithm is proposed and its effectiveness is shown by simulation experiments. A theoretical proof of convergence of the substrate control algorithm is given. A further advantage of the nonlinear approach of this paper is that the identified parameters (namely the growth rate and a yield coefficient) have a clear physical meaning and can give, in real time, a useful information on the state of the biomass.
\end{abstract}

\section{INTRODUCTION}

A COMMONLY used approach for the adaptive control of nonlinear systems is to consider them as time-varying linear systems and to use black-box linear approximate models to implement the control law. This approach has been used by the authors in previous works on the control of fermentation processes (Bastin and coworkers, 1983a, b).

But, since the underlying process is nonlinear, improved control can be expected by exploiting the nonlinear structure of the model. Such an idea is pursued in the present paper: we suggest how nonlinear adaptive control of nonlinear bacterial growth systems can be implemented. A similar idea has recently been used for the dissolved oxygen adaptive control in waste water treatment (Ko, McInnis and Goodwin, 1982), but under a somewhat different form than in the present paper.

\footnotetext{
* Received 20 October 1983; revised 20 March 1984. The original version of this paper was presented at the IFAC Workshop on Adaptive Systems in Control which was held in San Francisco, California, U.S.A. during June 1983. The published proceedings of this IFAC meeting may be ordered from Pergamon Press Ltd, Headington Hill Hall, Oxford OX3 $0 B W$, U.K. This paper was recommended for publication in revised form by associate editor P. Parks under the direction of editor B. Anderson.

† Laboratoire d'Automatique, de Dynamique et d'Analyse des Systèmes, University of Louvain, Bâtiment Maxwell, B-1348 Louvain-la-Neuve, Belgium.
}

The process is described by a nonlinear state space representation obtained from usual material balance equations (Section 2). However, this representation does not require any specific analytical description of the bacterial growth rate.

The system is then approximated by a discretetime time-varying model which is linear in the parameters and in the control input though globally nonlinear. The time-varying parameters in this model (namely the growth rate and a yield coefficient) have a clear physical meaning and are identified in real time with a standard RLS algorithm (Section 3).

The parameter estimation algorithm is combined with minimum-variance and Clarke-Gawthrop controllers to obtain adaptive controllers in two different cases: substrate concentration control (Section 4) and production rate control (Section 5). The effectiveness of the parameter estimation algorithm and the adaptive control algorithms is demonstrated by simulation experiments. Furthermore a theoretical proof of the convergence of the substrate control is given in the Appendix.

Parameter estimation and nonlinear control of microbial growth systems have been, in the last decade, the object of growing interest. Among many others, we may mention the papers by D'Ans, Kokotovic and Gottlieb (1971), Aborhey and Williamson (1978), Holmberg and Ranta (1982) and a large number of papers (and references) contained in the proceedings of the first IFAC Workshop on Modelling and Control of Biotechnical Processes (Halme, 1983), especially the contributions of Marsili-Libelli (1983) and Stephanopoulos and Ka-Yiu San (1983). However, we believe that the algorithms proposed in this paper have some original features that we can summarize as follows:

(a) In our approach, the parameter estimation and the process control are performed simultaneously. 
(b) The specific growth rate is not modelled by an analytical function of the state but is considered as a time-varying unknown parameter estimated in real time by a simple leastsquares algorithm.

(c) The control is performed by a very simple selftuning scheme which contrasts with more sophisticated approaches followed elsewhere like, e.g. nonlinear optimal control (D'Ans, Kokotovic and Gottlieb, 1971), nonlinear state feedback with Riemanian geometric model (Takamatsu, Shioya and Kurome, 1983) or adaptive multimodel control (Cheruy, Panzarella and Denat, 1983).

(d) Global convergence of the substrate control algorithm is established under mild conditions.

\section{DESCRIPTION OF THE SYSTEM}

We consider the usual state-space representation of bacterial growth systems by mass-balance equations

$$
\begin{aligned}
& \dot{X}=[\mu(X, S)-U] X \\
& \dot{S}=-k_{1} \mu(X, S) X+U(V-S) \\
& Y=k_{2} \mu(X, S) X
\end{aligned}
$$

with state variables: $X$ biomass concentration

$S$ substrate concentration

$\begin{array}{ll}\text { inputs: } & \begin{array}{l}U \text { dilution rate (i.e. influent } \\ \text { flow rate) }\end{array} \\ & V \text { influent substrate con- } \\ & \text { centration } \\ \text { outputs: } & S \text { substrate concentration } \\ & Y \text { production rate of the } \\ & \text { reaction product } \\ \text { parameters: } & \mu(X, S) \text { growth rate } \\ & k_{1} \text { and } k_{2} \text { yield coefficients. }\end{array}$

We could think of adopting an analytical expression for the bacterial growth rate $\mu(X, S)$; the most popular expression is certainly the Monod law

$$
\mu(X, S)=\frac{\mu^{*} S}{K_{M}+S}(\text { Monod })
$$

but many other expressions have been suggested, like

$$
\begin{gathered}
\mu(X, S)=\frac{\mu^{*}}{K_{\mathrm{b}}} S \quad S \leqslant K_{\mathrm{b}}(\text { Blackman) } \\
\mu^{*} \quad S \geqslant K_{\mathrm{b}} \\
\mu(X, S)=\frac{\mu^{*} S}{K_{\mathrm{c}} X+S} \quad \text { (Contois) } \\
\mu(X, S)=\frac{\mu^{*} K_{0} S}{1+K_{1} S+K_{2} S^{2}} \quad \text { (Haldane) }
\end{gathered}
$$

In these expressions, $\mu^{*}$ is the maximum growth rate.

The choice of an appropriate model for $\mu(X, S)$ is far from being an easy task and is the matter of continuing research (e.g. Roques and co-workers, 1982). Spriet (1982) lists no less than nine different models for $\mu(X, S)$ which have been proposed in the literature without even mentioning those which involve inhibitions (like the Haldane law (5)) or a $\mathrm{pH}$-dependence (e.g. Vandenberg and coworkers, 1976).

Furthermore, it is well known that important identifiability difficulties occur when estimating the parameters ( $\mu^{*}$ and $K_{\mathrm{m}}$ or $K_{\mathrm{b}}$ or $K_{\mathrm{c}} \ldots$ ) from real-life data (e.g. Holmberg and Ranta, 1982; Bastin and coworkers, 1983b, Holmberg, 1983).

Therefore we prefer to 'short-circuit' the problem of this choice and to identify the time-varying growth rate $\mu(X, S)$ in real-time by an adaptive algorithm.

Throughout this paper, we shall assume that:

(a) the dilution rate $U$ is the control input;

(b) the influent substrate concentration $V$ is an external measurable disturbance input;

(c) the substrate concentration $S$ and the production rate $Y$ are measurable outputs.

A typical example: the anaerobic digestion process

The state-space representation (1) is suited to describe the methanization stage in an anaerobic digestion process. The anaerobic digestion can be used, for instance, for the treatment of wastes in sugar industries: $U$ is the influent acetic acid concentration (i.e. the input pollution level), $S$ is the output pollution level and $Y$ is a methane gas flow rate. $V$ and $S$ are observed through BOD measurements. The main interest of such a water treatment plant is obviously to yield methane gas which can be used as an auxiliary energy supply Further details on the anaerobic digestion process can be found in Antunes and Installe (1981), Van den Heuvel and Zoetmeyer (1982), and Bastin and coworkers $(1983 \mathrm{a}, \mathrm{b})$.

\section{ADAPTIVE PARAMETER ESTIMATION}

Using a first-order Euler approximation for $\dot{X}$ and $\dot{S}$, with a sampling period $T$, the following discrete-time equations are derived from the system equations (1)

$$
\begin{aligned}
& X_{t+1}=X_{t}+T \mu_{t} X_{t}-T U_{t} X_{t}+\tilde{v}_{t} \\
& S_{t+1}=S_{t}-T k_{1} \mu_{t} X_{t}+T U_{t}\left(V_{t}-S_{t}\right)+\omega_{t} \\
& Y_{t}=k_{2} \mu_{t} X_{t}
\end{aligned}
$$

In these equations, the subscript $t$ is a discrete-time index $(t=0,1,2, \ldots)$ and the growth rate $\mu_{t}$ is a compact notation for $\mu_{t}=\mu\left(X_{t}, S_{t}\right)$. 
We make the approximation

$$
Y_{t+1}-Y_{t}=k_{2} \mu_{t}\left(X_{t+1}-X_{t}\right)+\varepsilon_{t} .
$$

Then, substituting for $X_{t}$ and $X_{t+1}$ from (7) into (6), we have

$$
\begin{gathered}
Y_{t+1}=Y_{t}+\mu_{t} T Y_{t}-T U_{t} Y_{t}+v_{t} \\
S_{t+1}=S_{t}+k T Y_{t}+T U_{t}\left(V_{t}-S_{t}\right)+\omega_{t}
\end{gathered}
$$

with

$$
\begin{aligned}
& v_{t}=\varepsilon_{t}+k_{2} \mu_{t} \bar{v}_{t} \\
& k=-\frac{k_{1}}{k_{2}} .
\end{aligned}
$$

Equations (8) and (9) constitute the basic discretetime model for the derivations of the parameter estimation and adaptive control algorithms. In this model, $v_{t}$ and $\omega_{t}$ represent errors due to noise, discretization and approximation (7).

Since the basic model is linear in the parameters $\mu_{t}$ and $k$, recursive least-squares estimates can be readily obtained

$$
\begin{gathered}
\hat{\mu}_{t+1}=\hat{\mu}_{t}+T P_{t} Y_{t}\left(Y_{t+1}-Y_{t}+T U_{t} V_{t}-\hat{\mu}_{t} T Y_{t}\right) \\
\hat{k}_{t+1}=\hat{k}_{t}+T P_{t} Y_{t}\left(S_{t+1}-S_{t}\right. \\
\left.-T U_{t}\left(V_{t}-S_{t}\right)-\hat{k}_{t} T Y_{t}\right) \\
P_{t}=\frac{P_{t-1}}{\lambda}\left(1-\frac{T^{2} Y^{2}{ }_{t} P_{t-1}}{\lambda+T^{2} Y^{2}{ }_{t} P_{t-1}}\right) \\
\text { with } P_{0} \gg 0 \text { and } 0<\lambda \leqslant 1 .
\end{gathered}
$$

$\lambda$ is a forgetting factor to allow the tracking of the time-varying growth rate $\mu_{t}$. This forgetting factor is also used for the estimation of the yield coefficient $k$ to allow for variations 'due to unobservable physiological or genetic events' (Holmberg and Ranta, 1982). Notice that the estimation of both parameters is decoupled but with a common gain $P_{t}$.

In addition to these parameter estimates, the biomass concentration $X$ can be estimated in realtime by writing $\hat{X}_{t}=Y_{t} / k_{2} \hat{\mu}_{t}$.

\section{Simulation results}

Simulation experiments have been performed using state equations (1) as the 'true' bacterial growth system, with a Monod growth rate (2). The following parameters and initial state values were used:

$$
\begin{array}{rr}
\mu^{*}=0.4 \quad K_{\mathrm{m}}=0.4 \quad k=-0.3636 \\
X_{0}=0.069 \quad S_{0}=0.13 .
\end{array}
$$

The initial values of both estimated parameters $\hat{\mu}_{t}$ and $\hat{k}_{t}$ were set to zero. These values will be used for all the simulation experiments throughout the paper. Figure 1 shows the estimates $\hat{\mu}_{t}$ and $\hat{k}_{t}$ computed by the algorithm equations (10) and (11) with $P_{0}=10^{6} \mathrm{I}$ and white noise input signals $U_{1}$ and $V_{t}$.

The same experiment is shown in Fig. 2, except that a jump is applied on the maximum growth rate $\left(\mu^{*}=0.4 \rightarrow 0.45\right)$ at time $t=240$.

We observe a fast convergence, without bias, of the parameter estimate $\hat{k}_{t}$ and a slower convergence of $\hat{\mu}_{t}$ to the 'true' time-varying growth rate $\mu_{t}$.

\section{SUBSTRATE CONTROL}

We consider the problem of regulating the substrate concentration $S_{t}$ at a prescribed level $S^{*}$ despite the disturbance input $V_{t}$, by acting on the dilution rate $U_{t}$.

In the anaerobic digestion example mentioned above, this is a depollution control problem with $V_{t}$ and $S_{t}$ the input and output pollution levels respectively.

A discrete-time minimum variance adaptive controller is adopted. At each sampling time, the control input $U_{t}$ is computed by setting a one-step ahead prediction of the substrate concentration equal to the prescribed level

$$
\hat{S}_{t+1}=S^{*}
$$

From the basic model equation (9), it is natural to define $\hat{S}_{t+1}$ as follows:

$$
\hat{S}_{t+1}=S_{t}+\hat{k}_{t} T Y_{t}+T U_{t}\left(V_{t}-S_{t}\right)
$$

here $\hat{k}_{t}$ is updated by the parameter estimation algorithm (11).

A nonlinear control law is readily obtained from (12), since $\hat{S}_{t+1}$ is linear in $U_{t}$; in practice, the control action $U_{t}$ is obviously constrained by the operating conditions. Therefore, the adaptive control algorithm is as follows:

$$
\begin{aligned}
& \bar{U}_{t}=\frac{S^{*}-S_{t}-\hat{k}_{t} T Y_{t}}{T\left(V_{t}-S_{t}\right)} \\
& U_{t}=0 \text { if } \bar{U}_{t}<0 \\
& \bar{U}_{t}=U_{\max } \text { if } \bar{U}_{t}>U_{\max } \\
& U_{t}=\bar{U}_{t} \text { otherwise. }
\end{aligned}
$$

A block diagram of the closed-loop system is presented in Fig. 3. We note that a feedforward compensation of the measurable perturbation $V_{t}$ is included.

\section{Simulation results}

Successful simulation experiments have been carried out, using the continuous-time state equations (1) as the 'true' system with a Monod growth rate (2) and $U_{\max }=0.39$. 

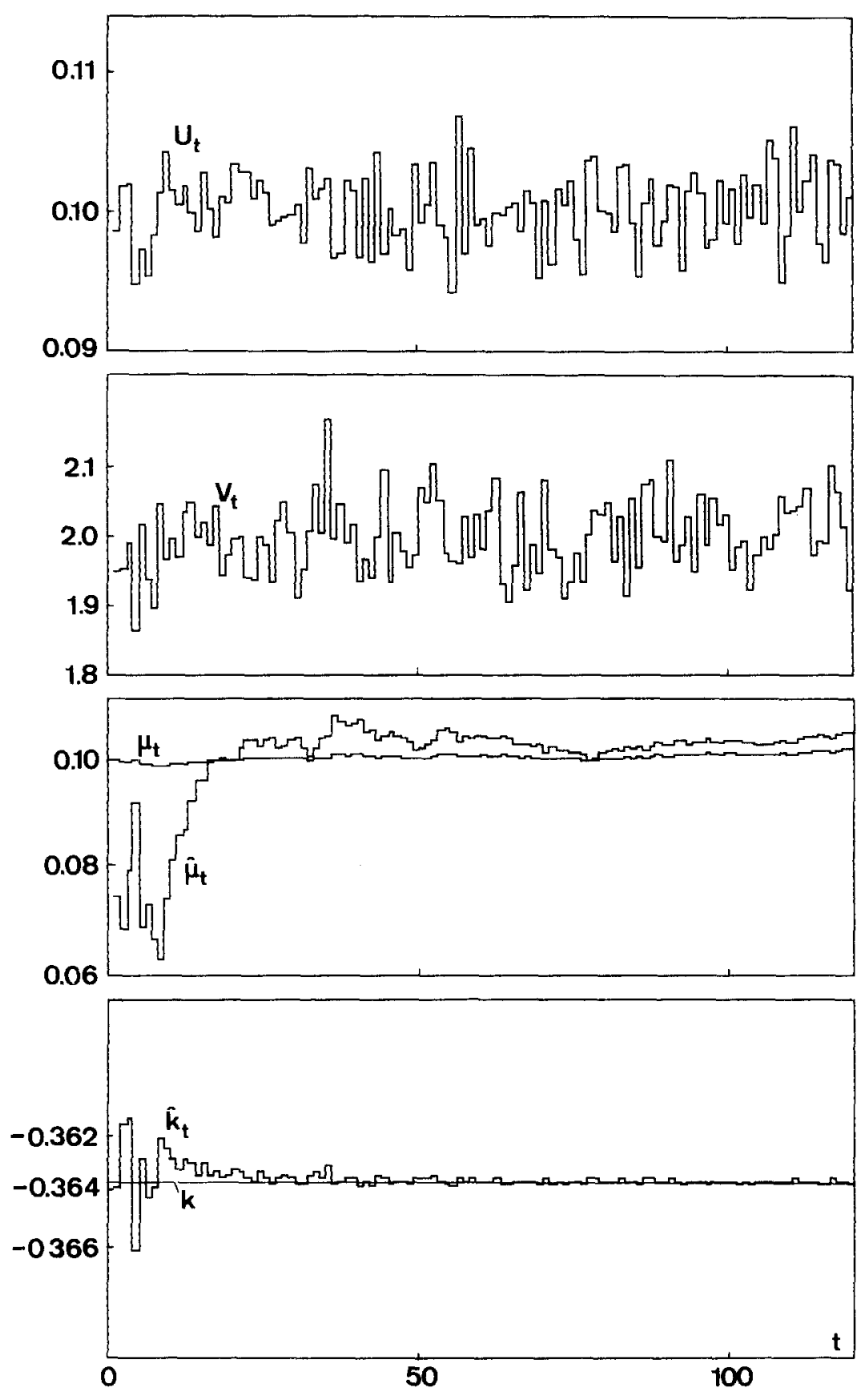

FIG. 1. Parameter estimation with white noise inputs.

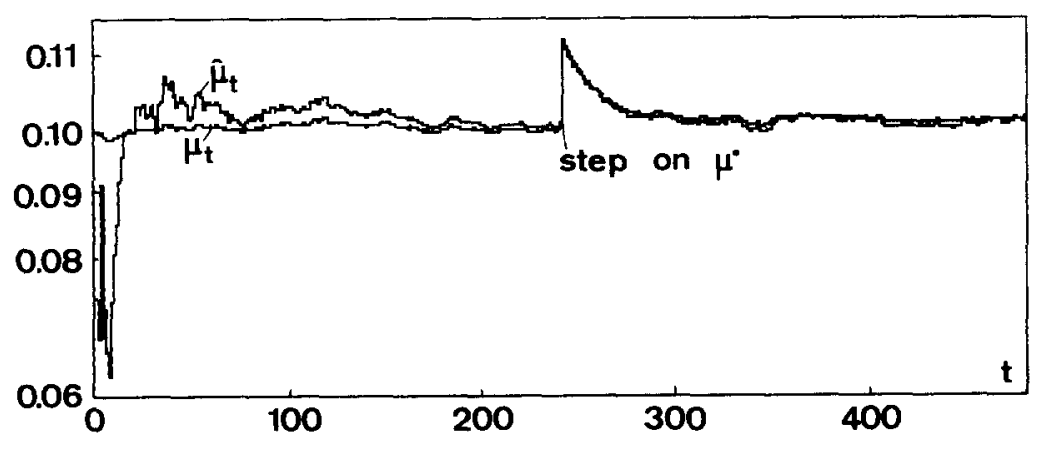

FIG. 2. Estimation of $\hat{\mu}_{t}$ with a step on $\mu^{*}$ at $t=240 \mathrm{~s}$. 


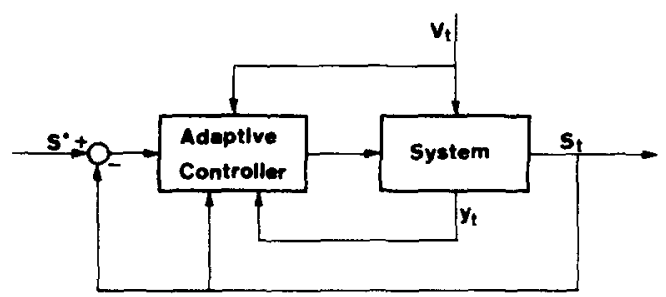

FiG. 3. Block diagram of the substrate concentration control.

Figure 4 shows the substrate concentration $S_{t}$, the control input $U_{t}$ and the parameter estimates $\hat{\mu}_{t}$ and $\hat{k}_{t}$ in the case of a square-wave set point with a period of 96 sampling times and a constant perturbation $V_{t}=2$. We observe that the controlled output $S_{t}$ converges much faster than the parameter estimate $\hat{\mu}_{t}$, but this is not surprising since $\hat{\mu}_{t}$ is not actually used by the control algorithm.

Figure 5 shows the substrate concentration $S_{t}$, the control input $U_{t}$ and the parameter estimates $\hat{\mu}_{t}$ and $\hat{k}_{t}$ in the case of a square-wave perturbation $V_{t}$ and an additive white noise on the auxiliary output $Y$. Evidently, we observe a bias (due to the noise) in the parameter estimates but this is not important for the convergence of controlled output $S_{t}$.

Figure 6 shows the substrate concentration $S_{t}$ and the control input $U_{t}$ in the case of a $10 \%$ squarewave variation of the maximum growth rate.
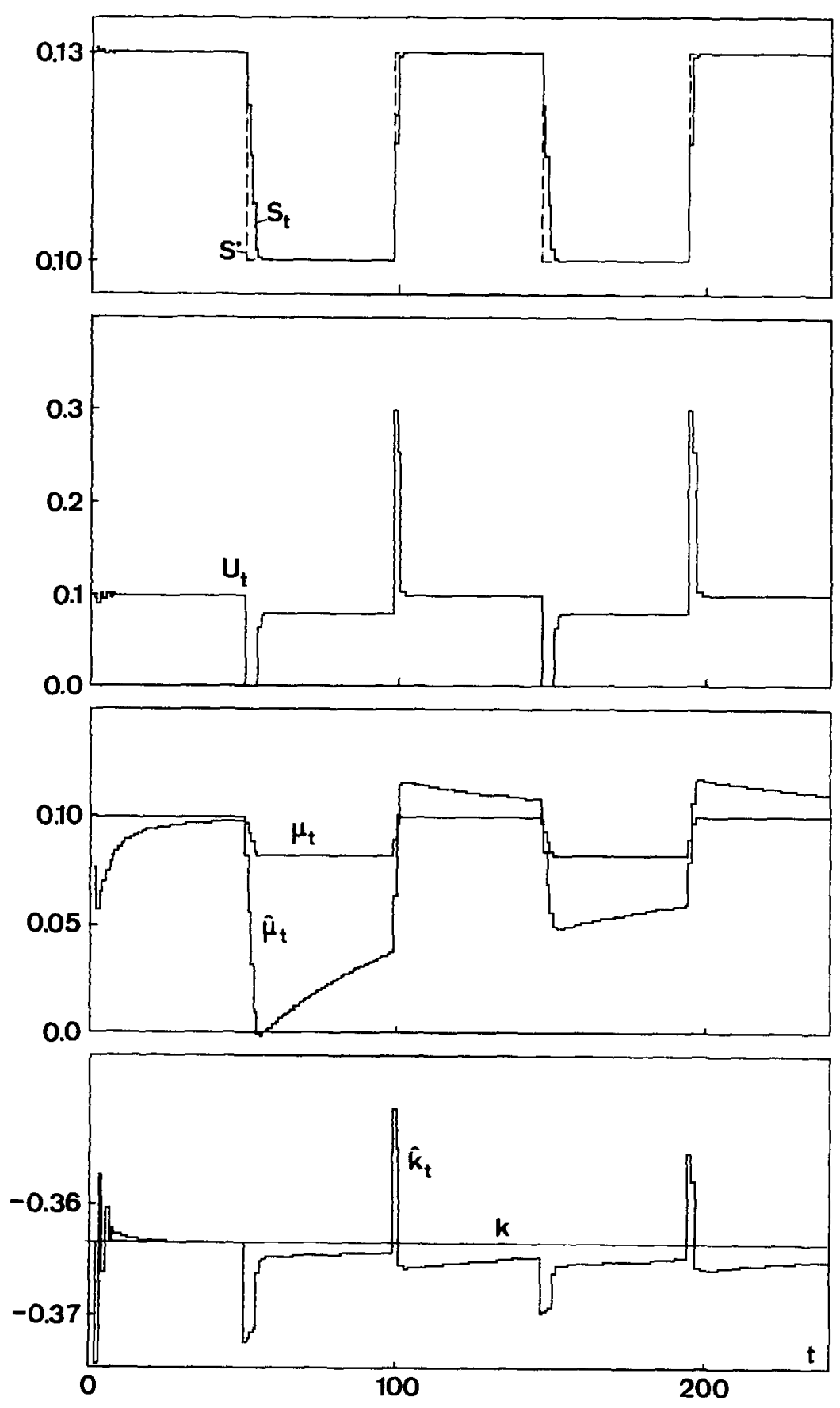

FIG. 4. Substrate concentration control with a square-wave set point. 

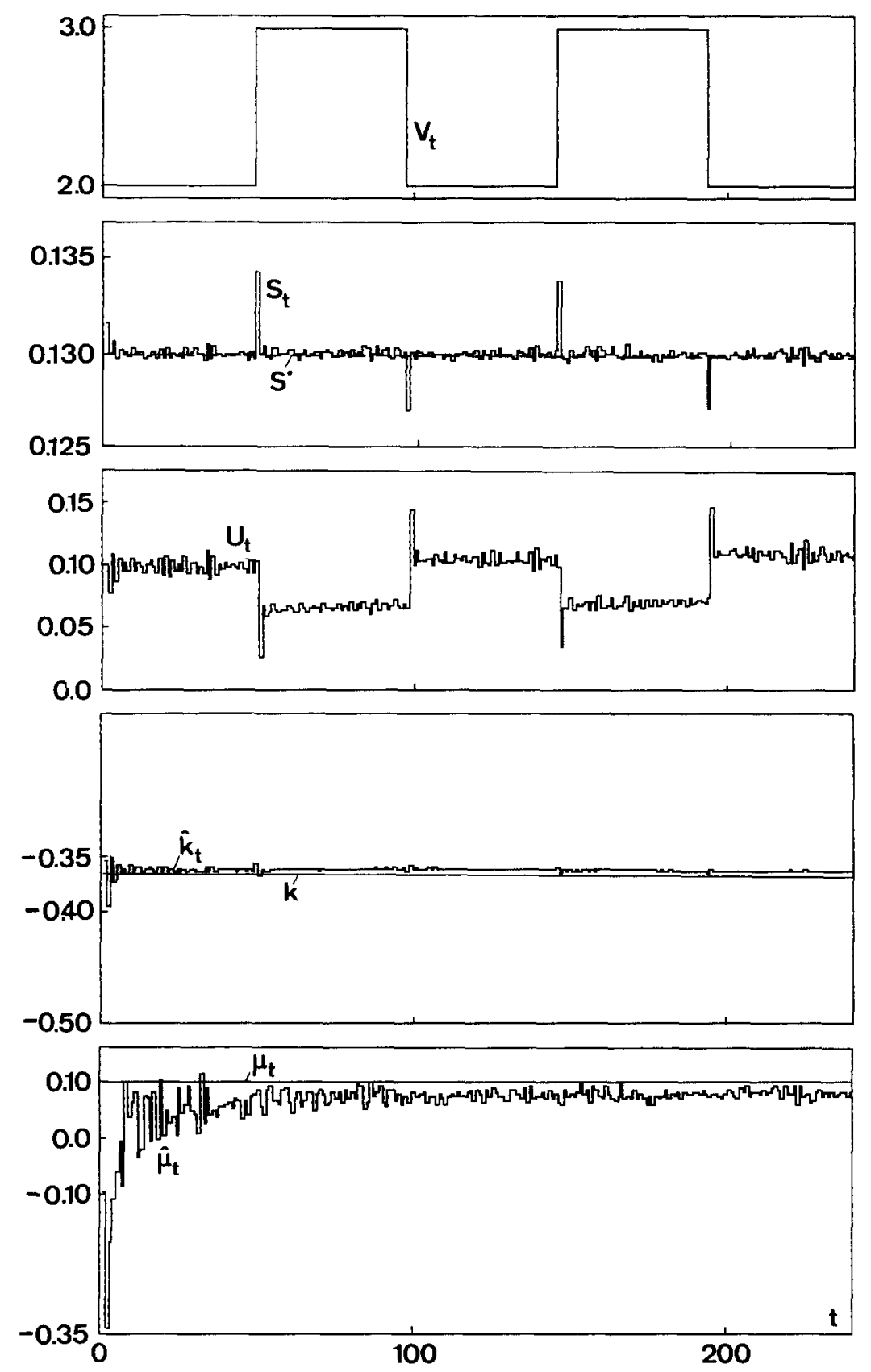

FIG. 5. Substrate concentration control with a square-wave input perturbation.

\section{PRODUCTION RATE CONTROL}

In order to facilitate the understanding of the later discussions, we refer here to the particular case of the anaerobic fermentation process described in Section 2 but, obviously, the results can also apply to other processes with the same structure.

The anaerobic digestion can be viewed as an energy conversion process. An amount of 'organic' energy is available in the influent under the form of the input organic load $V_{t}$. This energy is converted into methane gas $Y_{t}$ by the anaerobic digestion. Obviously, the output energy $Y_{t}$ cannot, in the mean, be larger than the available input energy. When the aim of the plant is not depollution but energy production (as in industrial farms), the control objective is to continuously adapt the output production $Y_{t}$ to the available input load $V_{t}$. Therefore, the desired gas production $Y_{t}^{*}$ is defined as follows:

$$
Y_{t}^{*}=\beta V_{t}-\beta_{0} \quad \beta>0, \quad \beta_{0}>0
$$

The coefficients $\beta$ and $\beta_{0}$ have to be selected carefully by the user since if, by lack of knowledge, $\beta$ is chosen too large or $\beta_{0}$ too small (i.e. if we require from the fermentor more methane gas than it can actually provide) then the process can be driven by the controller to a wash-out steady-state (Antunes 

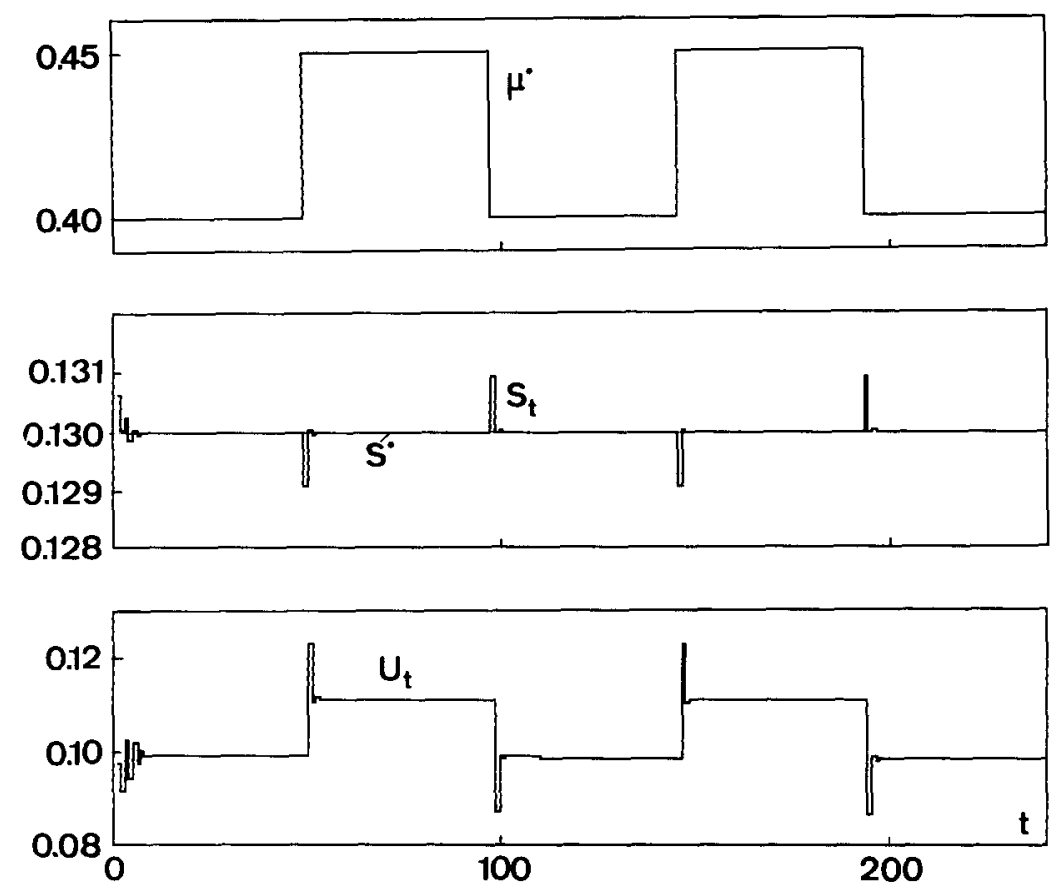

FIG. 6. Substrate concentration control with a square-wave perturbation on $\mu^{*}$.

and Installé, 1981), i.e. to a state where the bacterial life has completely disappeared and where the reactor is definitely stopped.

In this section, we shall first demonstrate that a minimum variance adaptive control law using the basic model equation (8) may diverge. We shall then describe what kind of modification we bring to the model in order to improve the control algorithm.

\section{Divergence of the minimum variance controller}

As for the substrate concentration control, we first try to use a minimum variance control law derived from the basic model equation (8)

$$
\begin{aligned}
\bar{U}_{t} & =-\frac{Y_{t+1}^{*}-Y_{t}\left(1+T \hat{\mu}_{t}\right)}{T Y_{t}} \\
U_{t} & =0 \text { if } \bar{U}_{t}<0 \\
U_{t} & =U_{\max } \text { if } \bar{U}_{t}>U_{\max } \\
U_{t} & =\bar{U}_{t} \text { otherwise. }
\end{aligned}
$$

Consider the case when $Y_{t+1}^{*}>Y_{t}\left(1+T \hat{\mu}_{t}\right)$. Then $\bar{U}_{t}<0$, i.e. $U_{t}$ is set to zero.

If $U_{t}$ is kept equal to zero, $Y_{t}$, possibly after a transient increasing period, will decrease and tend to zero (gas can no longer be produced if the influent has disappeared!). So, if the transient on $Y_{t}$ is not important enough, $U_{t}$ remains at the zero value, and $Y$ tends to zero.

Figure 7 illustrates this feature: at time $t=48$, the desired output level $Y_{t+1}^{*}$ is set to a value $15 \%$ larger than the steady-state value of $Y_{i}$.

\section{Modification of the basic discrete-time model}

In order to improve the control algorithm, we introduce the following modifications of the basic model equations.

First, we consider the following approximate relation between $\mu$ and $S$ :

$$
\mu(X, S)=b(X, S) . S
$$

i.e. the parameter $b$ is estimated, instead of $\mu$, with a recursive least-square algorithm.

One may consider this approximation as a loss of generality with respect to the previous case where $\mu$ is left independent of any analytical expression and estimated as a parameter of the system. But this is plainly justified by the fact that all the proposed bacterial growth laws are compatible with (17).

Rewrite the expression of $Y_{t}$, from (6)

$$
Y_{t}=k_{2} b_{t} S_{t} X_{t}
$$

We modify the approximation (7) by the following one:

$$
\begin{aligned}
Y_{t+1}-Y_{t}=k_{2} b_{t}[ & S_{t}\left(X_{t+1}-X_{t}\right) \\
& \left.+X_{t}\left(S_{t+1}-S_{t}\right)\right]+\epsilon_{t}
\end{aligned}
$$

i.e. the variation $\Delta Y_{t}=Y_{t+1}-Y_{t}$ is now dependent on both the variations $\Delta X_{t}$ in the bacterial 

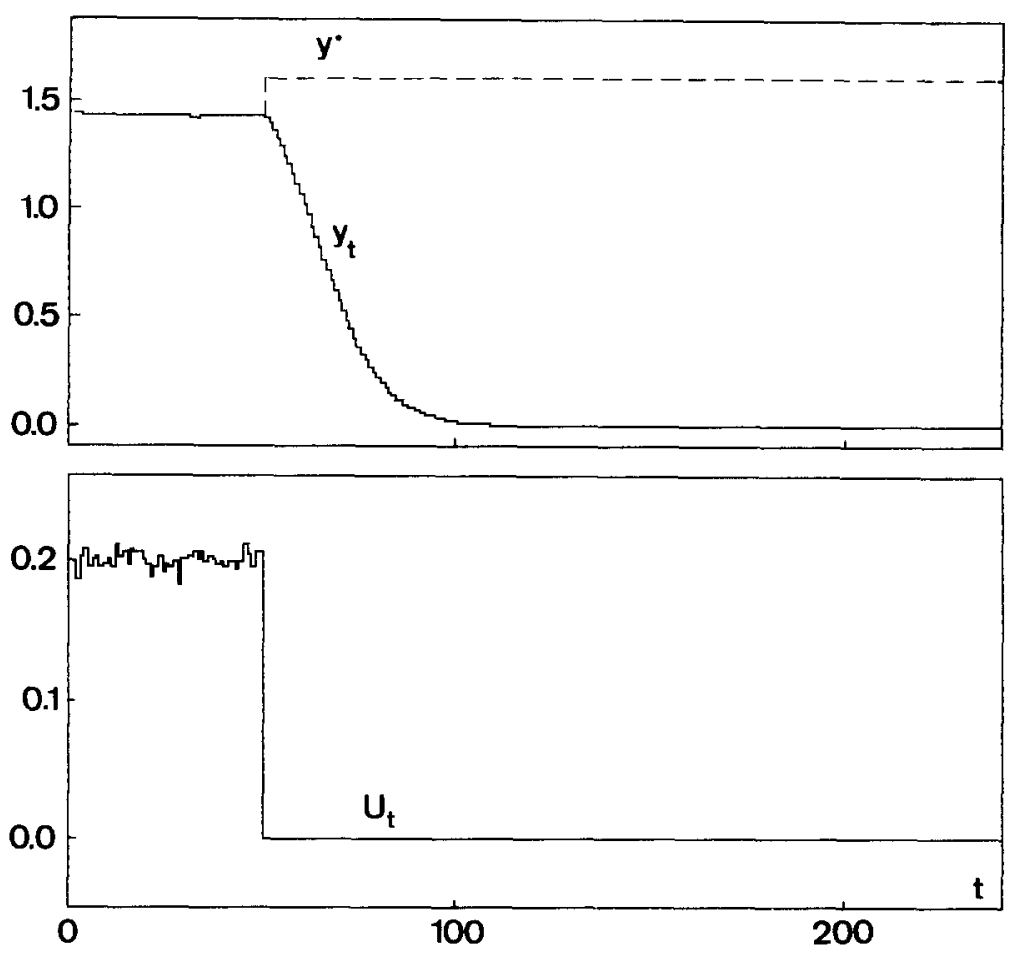

FIG. 7. Production rate control: divergence of a simple MV controller.

concentration and $\Delta S_{1}$ in the substrate concentration. Equation (8) becomes

$$
\begin{aligned}
Y_{t+1}=Y_{t}+b_{t} T S_{t} Y_{t} & +k T \frac{Y_{t}^{2}}{S_{t}} \\
& +T U_{t} Y_{t}\left(\frac{V_{t}}{S_{t}}-2\right)+v_{t}
\end{aligned}
$$

with $v_{t}=\varepsilon_{t}+k_{2} b_{t} S_{t} \tilde{v}_{t}+k_{2} b_{t} X_{t} \omega_{t}$.

Since (19) is linear in the parameter $b_{t}$, recursive least-squares estimates can be obtained

$$
\begin{aligned}
\hat{b}_{t+1}=\hat{b}_{t}+ & T S_{t} Y_{t} P_{t}^{\prime}\left(Y_{t+1}-Y_{t}-\hat{k}_{t} T \frac{Y_{t}^{2}}{S_{t}}\right. \\
& \left.+T U_{t} Y_{t}\left(\frac{V_{t}}{S_{t}}-2\right)-\hat{b}_{t} T S_{t} Y_{t}\right) \\
P_{t}^{\prime}= & \frac{P_{t-1}^{\prime}}{\lambda}\left(1-\frac{T^{2} Y_{t}^{2} S_{t}^{2} P_{t-1}^{\prime}}{\lambda+T^{2} Y_{t}^{2} S_{t}^{2} P_{t-1}^{\prime}}\right) .
\end{aligned}
$$

In these expressions the value of $\hat{k}_{\mathrm{t}}$ is assumed to be estimated by the recursive least-squares equation (11).

Notice that parameters $\hat{k}_{\mathrm{t}}$ and $\hat{b}_{t}$ are estimated in cascade'. This allows us to decouple the estimation of both parameters, and to keep a very simple scalar identification algorithm.

Figure 8 shows the same experiment as Fig. 1, but for the estimation of $\hat{b}_{t}$.

New minimum variance control algorithm

As above, we choose a discrete-time minimum variance adaptive controller. Using (19), the control input $U_{t}$ is given by

$$
\begin{aligned}
& \bar{U}_{t}=\frac{Y_{t+1}^{*}-Y_{t}-T \hat{k}_{t} Y_{t}^{2} / S_{t}-T \hat{b}_{t} S_{t} Y_{t}}{T Y_{t}\left(V_{t} / S_{t}-2\right)} \\
& U_{t}=0 \quad \text { if } \bar{U}_{t}<0 \\
& U_{t}=U_{\max } \text { if } \bar{U}_{t}>U_{\max } \\
& U_{t}=\bar{U}_{t} \quad \text { otherwise. }
\end{aligned}
$$

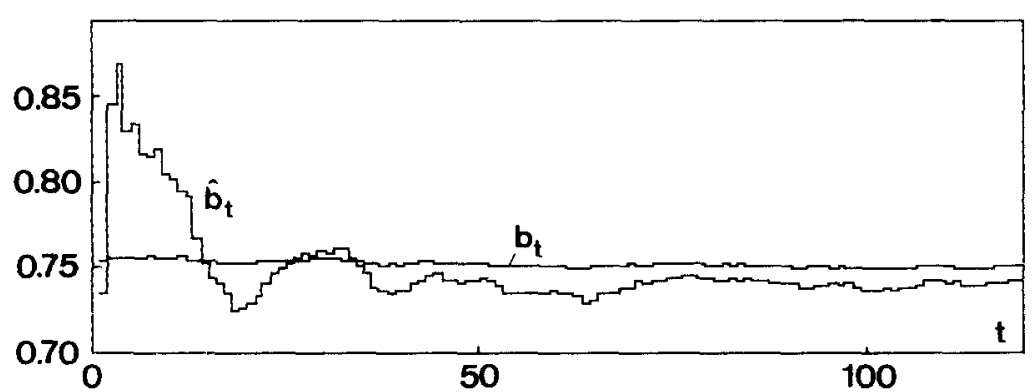

FIG. 8. RLS estimation of $b_{t}$. 


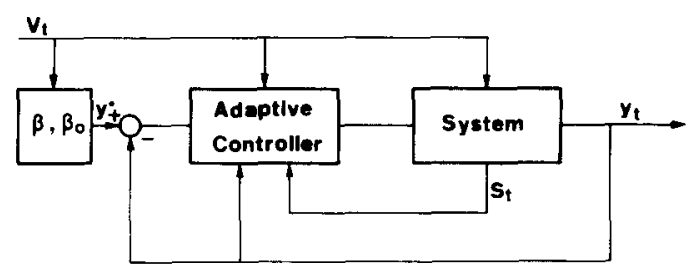

FIG. 9. Block diagram of the production rate control.

A block diagram of the closed-loop system is presented in Fig. 9.

\section{Simulation results}

The minimum variance adaptive controller, as written in (22), is more effective than the previous one (16). As a comparison, Fig. 10 shows the same experiment as Fig. 7, but with the control law (22).

In Fig. 11 steps of the influent substrate concentration $V_{t}$ (external measurable perturbation), i.e. of the desired output level $Y_{t}^{*}$ (see (15)), are applied to the system.

The control algorithm converges very quickly, although the convergence of the parameter $\hat{b}_{t}$ to its 'true' time-varying value is much slower.

\section{Clarke-Gawthrop controller}

It is evident, from (22), that the sign of $\bar{U}_{t}$ depends on the sign of $\left(V_{t} / S_{t}-2\right)$. When the substrate concentration $S_{\mathrm{t}}$ reaches values close to $0.5 V_{t}$, the minimum variance controller may appear not to be able to reach the desired set point. If $S_{t}$ is larger than $0.5 V_{t}, \bar{U}_{t}$ becomes negative, i.e. $U_{t}=0$. As a consequence, $S_{t}$ decreases. When $S_{t}$ becomes smaller than $0.5 V_{t}, \bar{U}_{t}$ is set to a positive value. If $\left(V_{t} / S_{t}-2\right)$ is close to zero, $\bar{U}_{t}$ most likely reaches large values, larger than $U_{\max }$, and $S_{t}$ increases again so that $\left(V_{t} / S_{t}-2\right)$ becomes negative, and so on.

In such a case, $U_{t}$ is oscillating between 0 and $U_{\text {max }}$, leading to the oscillation of the system, and the control does not converge. A typical illustration is given in Fig. 12.

In order to solve these convergence problems, we introduce a Clarke-Gawthrop (1979) control law using a weight $Q\left(1-z^{-1}\right)$ in the performance index (Belanger, 1983). The control input is then computed so as to minimize the following criterion:

$$
J=\left(\hat{Y}_{t+1}-Y_{t+1}^{*}\right)^{2}+Q^{2}\left(U_{t}-U_{t-1}\right)^{2} .
$$

Using (19), we have

$$
\begin{aligned}
& \bar{U}_{t}=\frac{Q^{2}}{Q^{2}+T^{2} Y_{t}^{2}\left(V_{t} / S_{t}-2\right)^{2}} U_{t-1} \\
& +\frac{T Y_{t}\left(V_{t} / S_{t}-2\right)}{Q^{2}+T^{2} Y_{t}^{2}\left(V_{t} / S_{t}-2\right)^{2}} \\
& {\left[Y_{t+1}^{*}-Y_{t}-\hat{k}_{t} T \frac{Y_{t}^{2}}{S_{t}}-\hat{b}_{t} T S_{t} Y_{t}\right] .}
\end{aligned}
$$

Figure 13 shows the improvement obtained by using this Clarke-Gawthrop controller. It is interesting to note that, as above, the convergence of the controlled output $Y_{t}$ (Fig. 13) is much faster than the convergence of the parameter estimates (Fig. 14).

\section{CONCLUSIONS}

Simple adaptive controllers for a class of biotechnical systems have been proposed. Their

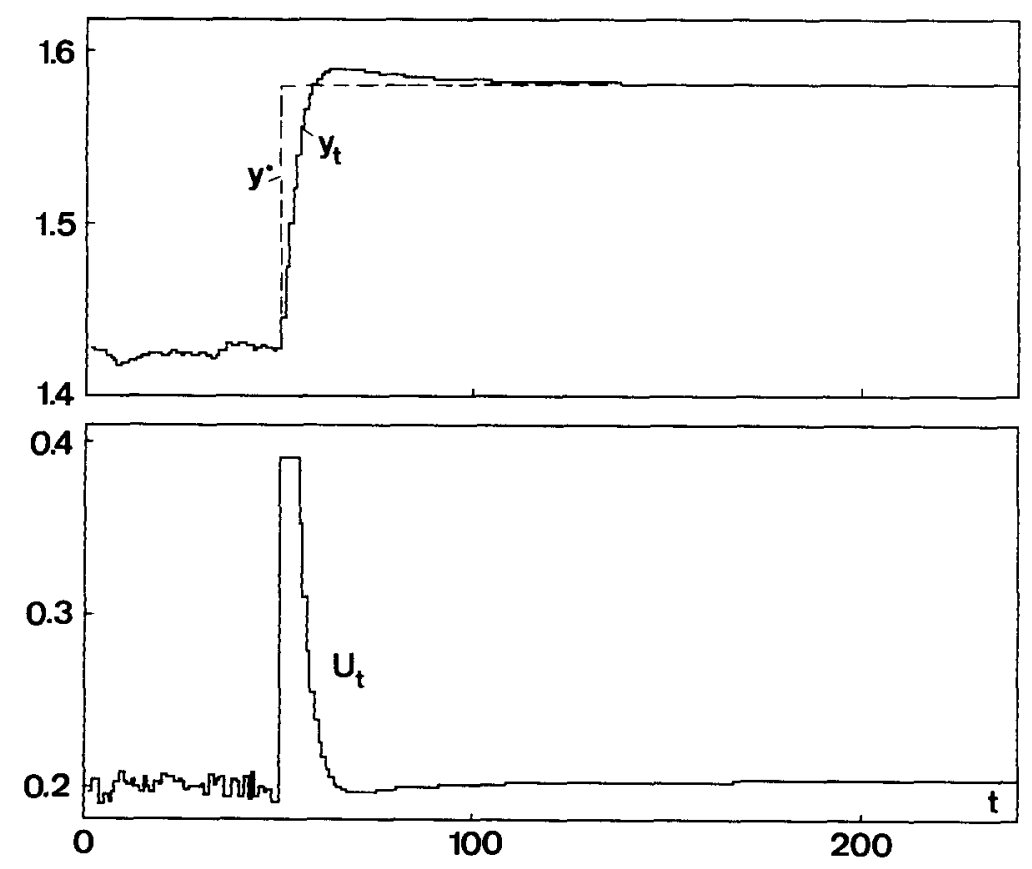

FIG. 10. Production rate control: convergence of the modified MV controller. 

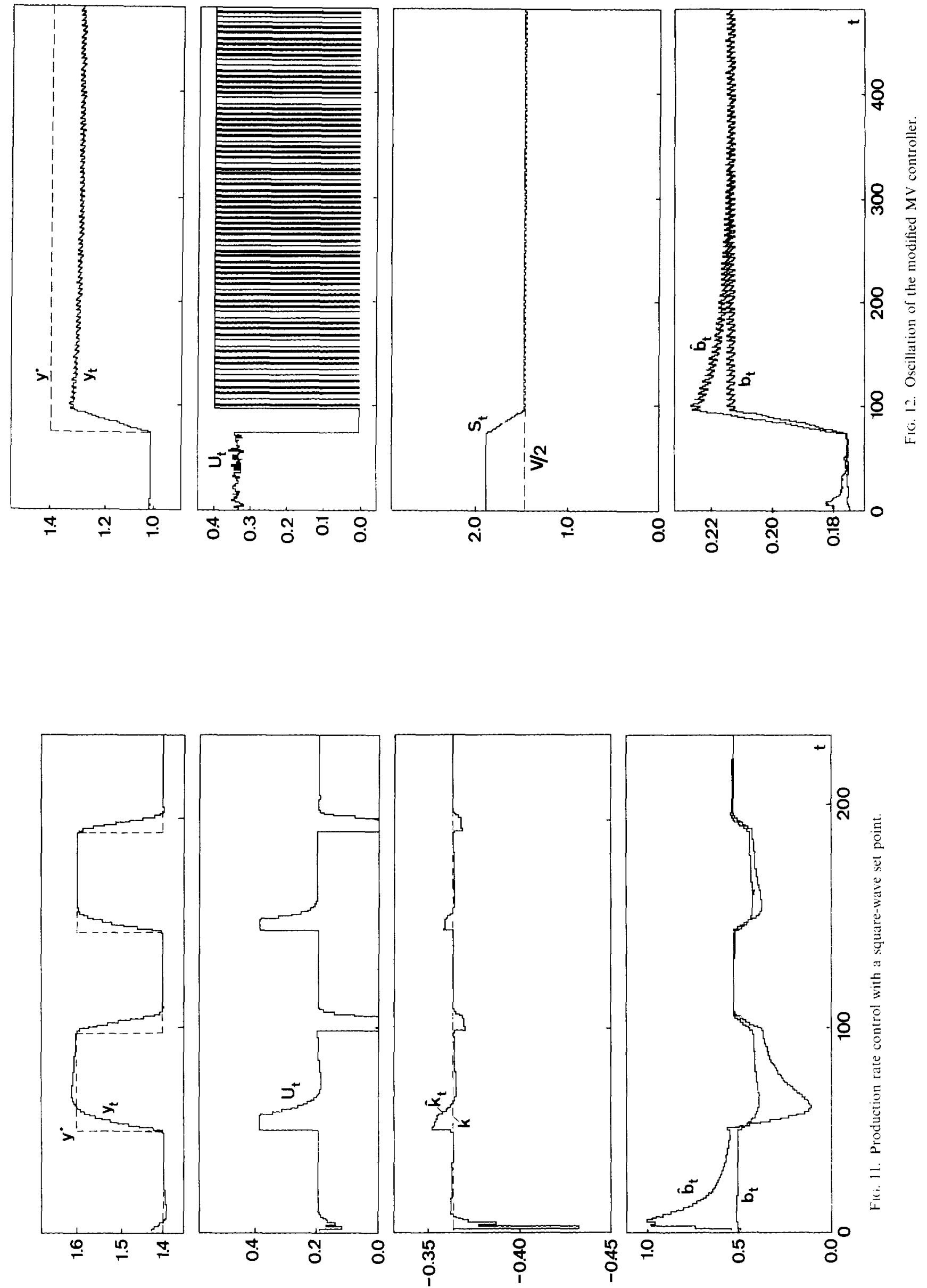

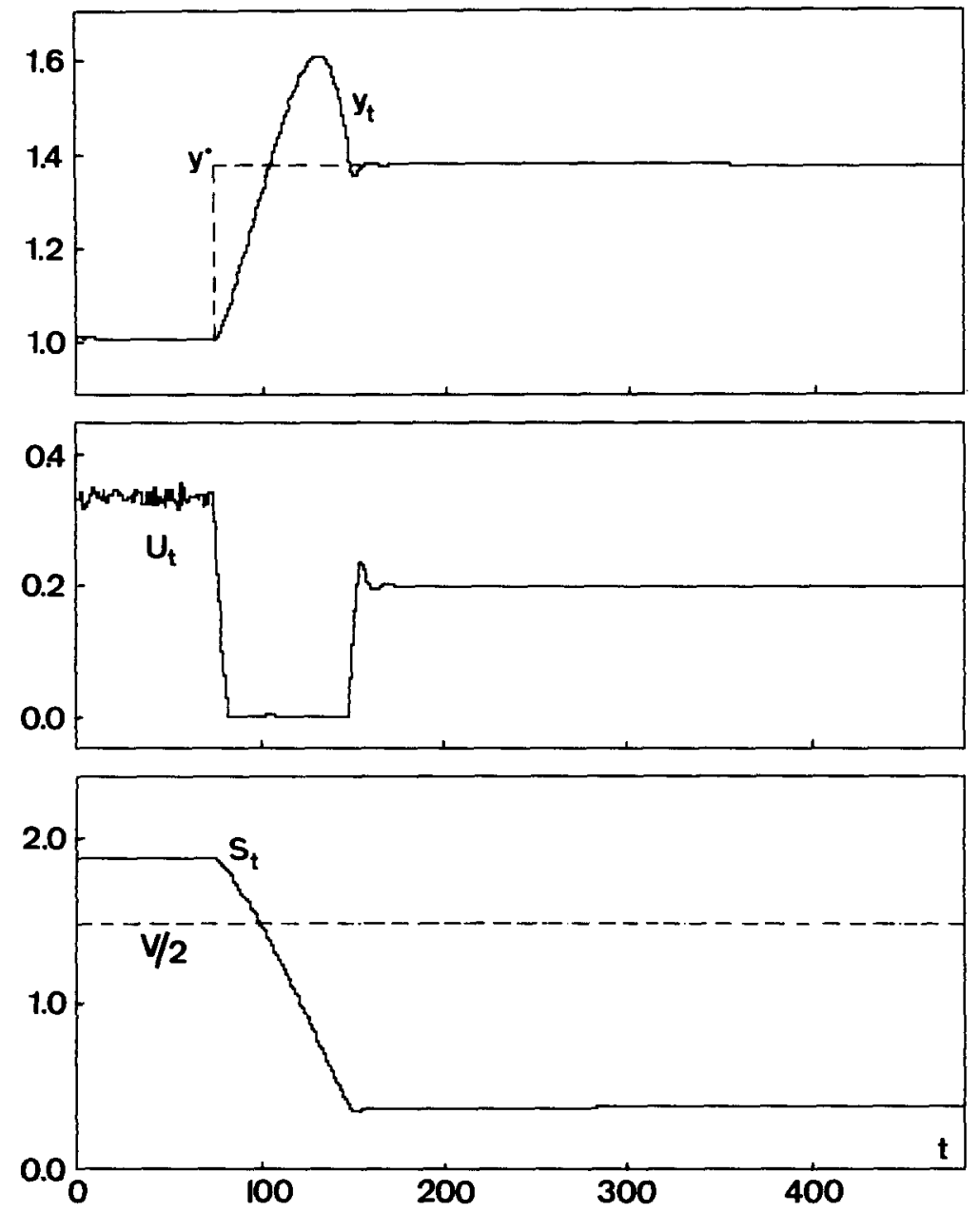

FiG. 13. Production rate control with a CG controller.
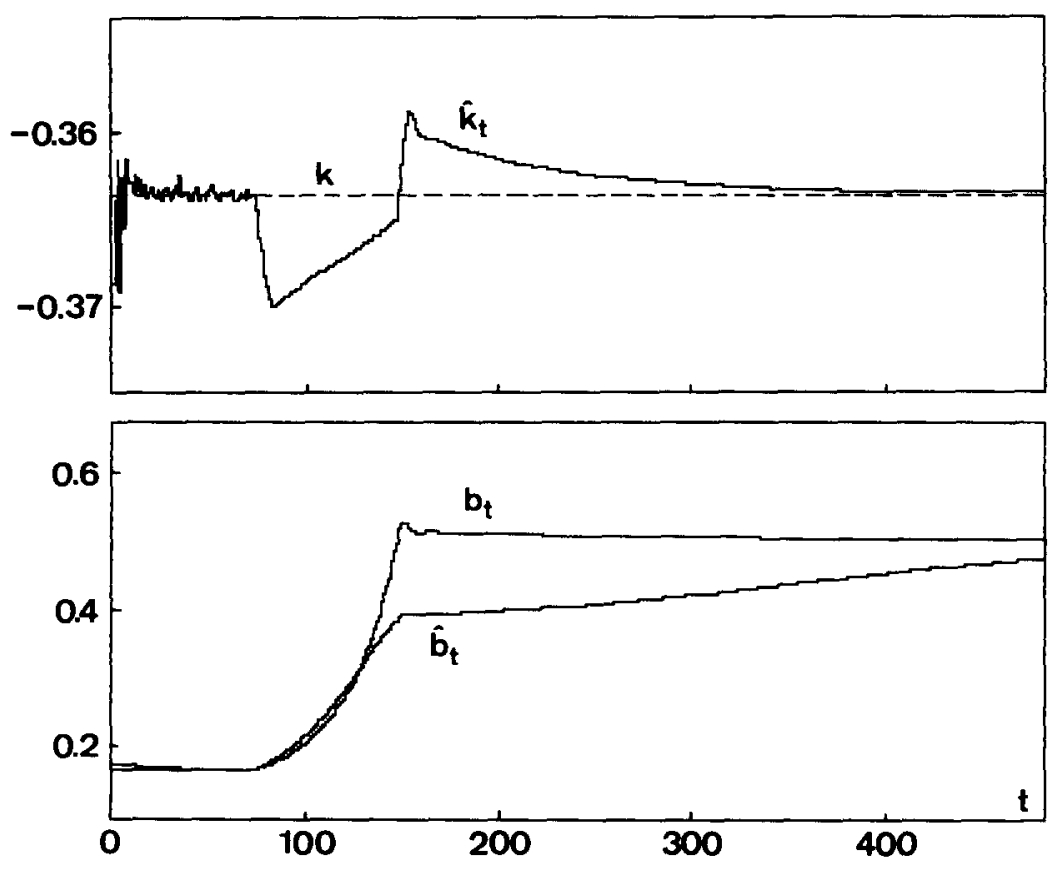

FIG. 14. Production rate control: evolution of the parameter estimates. 
effectiveness has been demonstrated by simulation experiments.

A theoretical proof of the convergence of substrate concentration control algorithm is given in the Appendix. In the case of production rate control, the convergence of the algorithm has not been discussed and is obviously much more difficult to establish since the algorithm involves two cascaded steps together with the estimation of a truly time-varying parameter $\left(\mu_{t}\right)$.

In addition to the control itself, a further advantage of the nonlinear approach of this paper is that the identified parameters correspond clearly to physical parameters (namely growth rate and yield coefficient); therefore they can provide useful information, in real-time, on the state of the biomass.

Although the model ( 1 ) is well suited to industrial applications like waste treatment in sugar industries, in many other applications the model (1) is only the last stage of a complex multistage reaction: a typical situation is a five-state twelve parameter model (e.g. Bastin and coworkers, 1983b) describing a sequence of three reactions (solubilization, acidification, methanization). This is a further reason to explore the possibility of simple control schemes for the different stages of such high-order highly nonlinear systems.

Acknowledgements - The authors wish to thank M. I. Installé from the Laboratory of Automatic Control (University of Louvain), H. Naveau, E. J. Nyns and D. Poncelet from the Laboratory of Biotechnology (University of Louvain), A. Cheruy and L. Dugard from the Laboratory of Automatic Control (ENSIEG, Grenoble) for fruitful discussions about this work.

\section{REFERENCES}

Aborhey, S. and D. Williamson (1978). State and parameter estimation of microbial growth processes. Automatica, 14, 493-498.

Antunes, S. and M. Installe (1981). The use of phase-plane analysis in the modelling and the control of a biomethanization process. Proceedings of the 8th IFAC World Congress, Kyoto, Japan, Vol. XXII, pp. 165-170.

Bastin, G., D. Dochain, M. Haest, M. Installé and P. Opdenacker (1983a). Modelling and adaptive control of a continuous anaerobic fermentation process. In Modelling and Control of Biotechnical Processes, A. Halme (ed). Pergamon Press, Oxford, pp. 299-306.

Bastin, G., D. Dochain, M. Haest, M. Installé and P. Opdenacker (1983b). Identification and adaptive control of a biomethanization process. In Modelling and Data Analysis in Biotechnology and Medical Engineering, G. C. Vansteenkiste and P. C. Young (eds). North-Holland, New York, pp. 271-282.

Belanger, P. R. (1983). On type I systems and the Clarke Gawthrop regulator. Automatica, 19, 91-94.

Cheruy, A., L. Panzarella and J. P. Denat (1983). Multimodel simulation and adaptive stochastic control of an activated sludge process. In Modelling and Control of Biotechnical Processes, A. Halme (ed). Pergamon Press, Oxford, pp. 127-183.

Clarke, D. W. and P. J. Gawthrop (1979). Self-tuning control. Proc. IEEE, 126, 633-640.

D'Ans, G., P. V. Kokotovic and D. Gottlieb (1971). A nonlinear regulator problem for a model of biological waste treatment. IEEE Trans. Aut. Control, AC-16, 341-347.
Goodwin, G. C., B. McInnis and R. S. Long (1982). Adaptive control algorithms for waste water treatment and $\mathrm{pH}$ neutralization. Opt. Control Appl. Meth. 3, 443-459.

Goodwin, G. C. and K. S. Sin (1983). Adaptive Filtering Prediction and Control. Prentice-Hall, Englewood Cliffs. NJ.

Halme, A. (1983). Modelling and control of biotechnical processes. Proceedings of the 1 st IFAC Workshop, Helsinki, Finland, 17-19 August 1982. Pergamon Press, Oxford.

Holmberg, A. (1983). On the accuracy of estimating in the parameters of models containing Michaelis-Menten type nonlinearities. In Modelling and Data Analysis in Biotechnology and Medical Engineering, G. C. Vansteenkiste and P. C. Young (eds). North-Holland, New York. pp. 199-208.

Holmberg, A. and J. Ranta (1982). Procedures for parameter and state estimation of microbial growth process models. Automatica, 13, 181-193.

Ko, K. Y.., B. C. McInnis and G. C. Goodwin (1982). Adaptive control and identification of the dissolved oxygen process. Automatica, 18, 727-730.

Marsili-Libelli, S. (1983). On-line estimation of bioactivities in activated sludge processes. In Modelling and Control of Biotechnical Processes, A. Halme (ed). Pergamon Press, Oxford, pp. 121-126.

Roques, H., S. Yve, S. Saipanich and B. Capdeville (1982). Is Monod's approach adaquate for the modelisation of purification processes using biological treatment? Water Resources, 16, 839-847.

Spriet, J. A. (1982). Modelling of the growth of micro-organisms: a critical appraisal. In Environmental Systems Analysis and Management, Rinaldi (ed). North-Holland, New York, pp. $451 \div 456$.

Stephanopoulos, G. and Ka-Yiu San (1983). On-line estimation of time-varying parameters: application to biochemical reactors. Modelling and Control of Biotechnical Processes, A. Halme (ed). Pergamon Press, Oxford, pp. 195-199.

Takamatsu T., S. Shioya and H. Kurome (1983). Dynamics and control of a mixed culture in an activated sludge process. Modelling and Control of Biotechinical Processes, A. Halme (ed). Pergamon Press, Oxford, pp. 103109.

Vandenberg, L., G. B., Patel, D. S. Clark and C. P. Lentz (1976). Factors affecting rate of methane formation from acetic acid by enriched methanogenic cultures. Can. J. Microbiol., 22 , 1312-1319.

Van den Heuvel, J. C. and R. J. Zoetmeyer (1982). Stability of the methane reactor: a simple model including substrate inhibition and cell recycle. Process Biochemistry. May-June, 14-19.

\section{APPENDIX: CONVERGENCE ANALYSIS OF THE SUBSTRATE CONCENTRATION CONTROL. ALGORITHM}

In this Appendix, we present a proof of the convergence of the substrate concentration control under a set of reasonable assumptions.

The demonstration has some similarities with that proposed by Goodwin, McInnis and Long (1982) in the case of dissolved oxygen control for waste water treatment.

It is organized in three steps:

(a) BIBO stability of the bacterial growth system,

(b) convergence of the parameter estimation algorithm,

(c) convergence of the adaptive control algorithm.

\section{BIBO stability of the continuous-time bacterial growth system}

Let us rewrite, for convenience, the state--space description of the bacterial growth system

$$
\begin{aligned}
& \dot{X}=[\mu(X, S)-U] X \\
& \dot{S}=-k_{1} \mu(X, S) X+U(V-S) \\
& Y=k_{2} \mu(X, S) X .
\end{aligned}
$$

In this section we prove the BIBO stability of this system (in accordance with the physical situation) under the following assumptions. 


\section{Assumptions.}

(H।a) $0 \leqslant \mu(X, S) \leqslant \mu^{*}$ (Hlb) $\mu(X, 0)=0$.

(H2a) $0 \leqslant U \quad 0 \leqslant V \leqslant V_{\max }$

(H2b) $0 \leqslant S(0) \quad 0 \leqslant X(0) \quad k_{1} X(0)+S(0) \leqslant V_{\text {max }}$.

Notice that all the growth rate models of Section 2 fulfill assumption $(\mathrm{H} 1)$.

Lemma 1. Under assumptions ( $\mathrm{H} 1)$ and $(\mathrm{H} 2)$

(i) $0 \leqslant S \leqslant V_{\max }$

(ii) $0 \leqslant X \leqslant \frac{V_{\max }}{k_{1}}$

(iii) $0 \leqslant Y \leqslant \frac{k_{2}}{k_{1}} \mu^{*} V_{\max } \forall t>0$.

Proof.

(I) $X \geqslant 0$ and $Y \geqslant 0$; straightforward by using (A la), (Alc) and (H2b).

(2) For $S=0$, we have $S \geqslant 0$, using ( $\mathrm{H} 2 \mathrm{a})$, (Alb) and ( $\mathrm{H} / \mathrm{b})$. The conclusion $S \geqslant 0$ for all $S$ follows from $(\mathrm{H} 2 \mathrm{~b})$.

(3) For $S=V_{\max }$, we have, using (Alb), (Alc) and (H2a).

$$
\dot{S}=-\frac{k_{1}}{k_{2}} Y+U\left(V-V_{\max }\right) \leqslant 0 \text { since } Y \geqslant 0 .
$$

The conclusion $S \leqslant V_{\max }$ for all $S$ follows from (H2b)

(4) Define the auxiliary variable $Z=k_{1} X+S$.

Then, the following equation is readily derived from (Ala) and $(\mathrm{A} 1 \mathrm{~b})$ :

$$
\dot{Z}=U(V-Z) .
$$

For $Z=V_{\max }$, we have $Z \leqslant 0$. The conclusion $Z \leqslant V_{\max }$ for all $Z$ follows. Since $S \geqslant 0$, clearly we have $X \leqslant V_{\max } / k_{1}$ for all $X$ and it becomes obvious that, by using (Alc) and (Hla),

$$
Y \leqslant \frac{k_{2}}{k_{1}} \mu^{*} V_{\max }
$$

Q.E.D.

It should be emphasized that from Lemma 1, the outputs $S$ and $Y$ and the state $X$ are bounded without imposing any upper bound on the input $U$.

\section{Convergence of the parameter estimation algorithm}

We consider now the convergence of the estimation algorithm for the parameter $\hat{k}_{t}$ presented in Section 3 .

The basic idea is due to Goodwin and Sin (1983) and can be roughly summarized as follows: if the noise term $\omega_{t}$ in (9) is bounded, then the convergence of the parameter estimate $\hat{k}_{t}$ can be guaranteed by involving, in the algorithm, a switching function to hold the parameter estimate constant wherever the prediction error becomes smaller than a prespecified bound. The algorithm (11) is considered without the forgetting factor $(\lambda=1)$ and modified as follows:

$$
\begin{gathered}
\hat{k}_{t+1}=\hat{k}_{t}+\sigma_{t} T P_{t} Y_{t}\left(S_{t+1}-\hat{S}_{t+1}\right) \\
P_{t}=P_{t-1}\left(1-\frac{\sigma_{t} T^{2} Y_{t}^{2} P_{t-1}}{1+\sigma_{t} T^{2} Y_{t}^{2} P_{t-1}}\right) P_{0}>0
\end{gathered}
$$

with $\hat{S}_{t+1}$ defined by (13)

\section{Assumptions.}

(H3) sup $\left|\omega_{t}\right| \leqslant \Delta$

$$
\text { (H4) } \begin{aligned}
\sigma_{t} & =1 \text { if } \frac{\left(S_{t+1}-\hat{S}_{t+1}\right)^{2}}{1+T^{2} Y_{t}^{2} P_{t-1}}>\Delta^{2} \\
\sigma_{t} & =0 \text { otherwise. }
\end{aligned}
$$

Lemma 2. For the algorithm (A6) and (A7), subject to assumptions $(\mathrm{H} 1)-(\mathrm{H} 4)$, then

$$
\lim _{t \rightarrow \infty} \sup \left|S_{t}-\hat{S}_{t}\right| \leqslant C \Delta
$$

with $C$ a positive constant independent of $\Delta$

\section{Proof.}

Let $\bar{k}_{t}=\hat{k}_{t}+\frac{k_{1}}{k_{2}}$.

Then, the following expression is readily derived from (A6) and (13):

$$
\frac{\tilde{k}_{t+1}}{P_{t}}=\frac{\tilde{k}_{t}}{P_{t-1}}+\sigma_{t} T Y_{t} \omega_{t}
$$

Then, by assumption (H4),

$$
\frac{\hat{k}_{t+1}^{2}}{P_{t}}-\frac{\hat{k}_{t}^{2}}{P_{t-1}} \leqslant\left[\Delta^{2}-\frac{\left(S_{t+1}-\hat{S}_{t+1}\right)^{2}}{1+\sigma_{t} T^{2} Y_{t}^{2} P_{t-1}}\right] .
$$

Then, $\frac{\tilde{k}_{t}^{2}}{P_{t-1}}$ is a nonincreasing function, bounded below by zero (since $P_{t-1}>0$ ) and

$$
\lim _{t \rightarrow \infty} \sigma_{t}\left[\Delta^{2}-\frac{\left(S_{t+1}-\hat{S}_{t+1}\right)^{2}}{1+\sigma_{t} T^{2} Y_{t}^{2} P_{t-1}}\right]=0
$$

Hence $\lim _{t \rightarrow \infty} \sup \left[\frac{\left(S_{t+1}-\hat{S}_{t+1}\right)^{2}}{1+\sigma_{t} T^{2} Y_{t}^{2} P_{t-1}}\right] \leqslant \Delta^{2}$.

Now, from (A7), the sequence $P_{t}$ converges and we define

$$
P_{\infty}=\lim _{t \rightarrow \infty} P_{t} \geqslant 0 .
$$

Hence, in view of Lemma 1 ,

$$
\lim _{t \rightarrow \infty} \sigma_{t} Y_{t}^{2} P_{t-1} \leqslant T^{2}\left(\frac{k_{2}}{k_{1}} \mu^{*} V_{\max }\right)^{2} P_{\infty}=C^{2}-1
$$

and, from (A15),

$$
\lim _{t \rightarrow \infty} \sup \left|S_{t+1}-\hat{S}_{t+1}\right| \leqslant C \Delta .
$$

\section{Convergence of the adaptive control algorithm}

We consider now the adaptive control algorithm (14). We have the following convergence result.

\section{Theorem.}

If (i) $V_{\min } \leqslant V \leqslant V_{\max }$

(ii) $S^{*}<V_{\text {min }}$

(iii) the parameter estimation algorithm (A6) and (A7) and the adaptive control algorithm (14) are used with

$$
U_{\max } \geqslant \frac{\mu^{*} V_{\max }}{V_{\min }-S^{*}}
$$

(iv) Assumptions (H1), (H2b), (H3) and (H4) hold

then $\lim \sup \left|S_{t}-S^{*}\right| \leqslant C \Delta$

with $C$ the same constant as in Lemma 2 . 
Proof. From Lemma 2, for each $\eta>0$, there exists $t_{0}>0$ such that

$$
\hat{S}_{t}-C \Delta-\eta \leqslant S_{\mathrm{t}} \leqslant \hat{S}_{\mathrm{t}}+C \Delta+\eta \text { for all } t \geqslant 0 .
$$

Define the interval

$$
I=\left[S^{*}-C \Delta-\eta, S^{*}+C \Delta+\eta\right] .
$$

(1) If the control algorithm gives $0<U_{t 0}<U_{\max }$ then $\hat{S}_{\mathrm{t}_{0}+1}$ $=S^{*}$ and hence $S_{t_{0}+1} \in I$.

(2) If the control algorithm gives $U_{t}=0$ for $t=t_{0}+k$ for $k=0,1,2,3, \ldots$ then by definition of $\bar{U}_{t}$, we have $\hat{S}_{t_{0}+k} \geqslant S^{*}$ for $k=1,2,3, \ldots$ and hence $S_{t_{0}+k} \geqslant S^{*}-C \Delta-\eta$.

But, if $U_{t}=0, S_{\mathrm{t}}$ decreases and tends asymptotically to the steady-state $S=0$, and there exists $k^{\prime}$ such that $S_{t_{0}+k^{\prime}} \leqslant S^{*}+C_{3} \Delta+\eta$. The conclusion $S_{t_{0}+k^{\prime}} \in I$ follows. If the sequence $U_{t_{0}+k^{\prime}}=0$ terminates at time $t^{\prime}>t_{0}$ so that $S_{t^{\prime}} \in I$ and $0<U_{t^{\prime}}<U_{\max }$, then $S_{t^{\prime}+1} \in I$ as in (1) above.

(3) If the control algorithm gives $U_{t}=U_{\max }$ for $t=t_{0}+k$, $k=0,1,2,3, \ldots$ then $S_{1}$ increases since $\dot{S} \geqslant 0$ by definition of $U_{\max }$ and we can prove similarly that $S_{t_{0}+k^{\prime}} \in I$ or $S_{t^{\prime}+1} \in I$.

(4) So far, we have shown that there exists some $t_{1}>t_{0}$ such that $S_{t_{1}} \in I$. Now it is easy to show that, if $S_{t,} \in I$, then $S_{t} \in I$ for all $t \geqslant t_{1}$, by using the arguments of $(1)-(3)$ above.

Thus we have

$$
\left|S_{i}-S^{*}\right| \leqslant C \Delta+\eta \text { for all } t \geqslant t_{1}
$$

Since $\eta>0$ may be chosen arbitrarily small, it follows from the definition of $l$ :

$$
\lim _{i \rightarrow \infty} \sup \left|S_{i}-S^{*}\right| \leqslant C \Delta
$$

\section{Comments.}

(1) The controller achieves a zero steady-state error even with a varying disturbance $V$, since the algorithm includes feedforward compensation.

(2) In order to prove the convergence, the switching function $\sigma_{t}$ has been included in the control algorithm and the following assumption has been stated

$$
U_{\max } \geqslant \frac{\mu^{*} V_{\max }}{V_{\min }-S^{*}} .
$$

It is worth noting that these precautions were omitted in the simulation results presented above, since $U_{\max }$ was arbitrarily fixed at 0.39 and the switching function $\sigma_{t}$ was not used in practice. These are necessary to prove the theoretical results but appear to be usually inoperative in the simulation experiments. 